\title{
Evaluation of Early Markers of Nephropathy in Patients with Type 2 Diabetes Mellitus
}

\author{
Pierina De Muro, ${ }^{1}$ Antonio Junior Lepedda, ${ }^{1}$ Gabriele Nieddu, ${ }^{1}$ Michela Idini, \\ Hai Quy Tram Nguyen, ${ }^{1}$ Omar Lobina, ${ }^{1}$ Pietro Fresu, ${ }^{2}$ and Marilena Formato ${ }^{1}$ \\ ${ }^{1}$ Department of Biomedical Sciences, University of Sassari, 07100 Sassari, Italy \\ ${ }^{2}$ Department of Diabetology and Metabolic Disease, AOU-Sassari, 07100 Sassari, Italy
}

Correspondence should be addressed to Pierina De Muro; demuro@uniss.it and Marilena Formato; formato@uniss.it

Received 13 November 2015; Revised 28 December 2015; Accepted 10 January 2016

Academic Editor: Tzi B. Ng

Copyright ( 2016 Pierina De Muro et al. This is an open access article distributed under the Creative Commons Attribution License, which permits unrestricted use, distribution, and reproduction in any medium, provided the original work is properly cited.

\begin{abstract}
Aims. T2DM often remains undiagnosed for many years because hyperglycemia develops gradually and may not produce any symptoms. As patients with T2DM are at increased risk of microvascular and macrovascular complications, the preclinical diagnosis of the state is the key point of the disease management. Methods. We evaluated parameters such as GAGs/PGs, NAG, and NGAL in urine samples from 43 normoalbuminuric T2DM patients and 31 apparently healthy control subjects. Results. The total urinary GAG excretion showed no significant differences between patients and controls. The electrophoretic analysis evidenced the presence of UTI and its degradation products (LSC and SM-LSC), CS, and HS. We observed modifications of HS and total UTI (including UTI and its degradation products) relative contents in T2DM patients compared with controls whereas no differences in CS percentage were found. NGAL levels were significantly increased in T2DM patients and were positively correlated with both NAG $(r=0.606$, $p<0.0001)$ and the presence of hypertension $(r=0.352, p<0.05)$. Conclusions. These data suggest that the assessed molecules could represent useful markers to detect early renal impairment in patients with T2DM.
\end{abstract}

\section{Introduction}

The chronic noncommunicable diseases represent one of the most difficult challenges for all health care systems, in both industrialized and developing countries, due to their continuous and relentless growth. The most paradigmatic example is certainly represented by diabetes mellitus. Epidemiological evidences suggest that, without effective prevention and control plans, diabetes will likely continue to increase globally [1]. In particular, T2DM often goes undiagnosed for many years because hyperglycemia develops gradually and may not produce any symptoms [2]. Since patients with diabetes are at increased risk of microvascular and macrovascular complications [3], the preclinical diagnosis of the state is the key point of the disease management.

The renal impairment in diabetes mellitus affects $~ 40 \%$ of type 1 and type 2 diabetic patients. Diabetic nephropathy is responsible not only for ESRD, but also for a significant increase in cardiovascular risk in this population. Although
CKD is a common comorbidity condition of T2DM, in the early stages it is often unrecognized, especially in the elderly, and, therefore, untreated [4]. The high incidence of CKD highlights the importance of early diagnosis and treatment for delaying its progression [5]. Because of the increasing T2DM prevalence worldwide and the serious consequences of this disease on global health, it would be of great value to develop more useful and reliable (and less expensive) diagnostic tests. Theoretically, urine represents an excellent substrate for identifying both potential biomarkers for early diagnosis of nephropathy and more effective therapeutic targets or monitoring the progression of renal damage. UAE is currently the "gold standard" for detection or prediction of both diabetic nephropathy and cardiovascular risk, even though in T2DM its predictive power is probably limited to cardiovascular events rather than renal functional impairment. In addition, structural changes in the GBM may occur before the onset of microalbuminuria. In the early stages of diabetic nephropathy, characterized by 
a low urinary excretion of albumin, the charge-dependent glomerular permselectivity seems to be particularly affected [6]; these findings suggest an initial loss of functional groups in the GBM with a consequent increase in the pore size of the renal filtration barrier. This charge-dependent permeability of the GBM is probably due to the presence of anionic constituents, especially HSPG. It is therefore important to identify urinary markers that may offer greater sensitivity, earlier detection, and greater predictive power for diabetes complications to overcome limits of UAE tests.

Over the past few years we have described quali-quantitative variations of GAGs/PGs in both normoalbuminuric type 1 and 2 diabetic patients with respect to healthy control subjects, suggesting that both levels and relative abundance of urinary GAGs may be predictive of altered GAG metabolism in diabetes mellitus $[7,8]$. Furthermore, we recently evidenced that UTI, a small PG found in urine, may represent a useful marker for monitoring kidney function in those patients at high risk of developing renal impairment [9].

It has been shown that tubular damage occurs early in the course of diabetic nephropathy and that it is not merely secondary to glomerular damage as previously thought [10]. From a clinical point of view, the excretion of tubular markers may have a higher predictive power compared to UAE. In particular, the concentration of NAG may even increase in the absence of elevated albumin excretion. In addition, NGAL is one of the earliest expressed molecules by renal tubular cells after insults of various origin. An increase of both serum and urinary NGAL has been described in diabetic patients before the onset of microalbuminuria, corroborating the recent theories of a phase of subclinical tubular involvement that anticipates the future appearance of the most well-known glomerular dysfunction [11].

Therefore, the aim of the present study is to assess parameters such as urinary GAGs/PGs, NAG, and NGAL to detect alterations of renal function in normoalbuminuric patients with T2DM.

\section{Materials and Methods}

2.1. Subjects. 43 normoalbuminuric patients with type 2 diabetes mellitus, referred to the Unità Operativa di Diabetologia e Malattie del Ricambio, AOU-Sassari, were enrolled. Exclusion criteria were ketoacidosis, fever, infection, surgery as well as evidences of systemic diseases, renal, cardiac, or hepatic diseases, and malignant tumors. Normoalbuminuria was defined as a urinary albumin excretion rate lower than $30 \mathrm{mg} / 24 \mathrm{~h}$ (mean of three different samples over a period of three months). The mean age of the T2DM patients included (16 men and 27 women) was $64.21 \pm 7.18$ years and the mean value of known duration of diabetes was $7.02 \pm 5.15$ years. The patients had no complications at the time of the study, but 27 of them (62.8\%, 8 men and 19 women) were hypertensive (systolic blood pressure $\geq 160 \mathrm{mmHg}$ and/or diastolic blood pressure $\geq 90 \mathrm{mmHg}$ ) and were under antihypertensive treatment. Table 1 shows the demographic and clinical characteristics of patients.

The control group was composed of 31 apparently healthy subjects matched for age, sex, and BMI with T2DM patients.
TABLE 1: Demographic and clinical characteristics of both patients and controls.

\begin{tabular}{lcc}
\hline Parameters & Controls & Patients \\
\hline Age (years) & $61.14 \pm 6.65$ & $64.21 \pm 7.18$ \\
Sex (men/women) & $10 / 21$ & $16 / 27$ \\
BMI $\left(\mathrm{kg} / \mathrm{m}^{2}\right.$ ) & $26.355 \pm 2.247$ & $27.583 \pm 3.619$ \\
Duration of disease (years) & & $7.02 \pm 5.15$ \\
Blood glucose (mg/dL) & $88.26 \pm 21.21$ & $131.59 \pm 33.40$ \\
HbAlc $(\%)$ & & $6.34 \pm 0.73$ \\
Total cholesterol (mg/dL) & $165.35 \pm 24.38$ & $170.71 \pm 27.01$ \\
HDL cholesterol (mg/dL) & $49.22 \pm 10.21$ & $55.20 \pm 11.20$ \\
LDL cholesterol (mg/dL) & $92.54 \pm 13.58$ & $96.24 \pm 18.91$ \\
Triglycerides (mg/dL) & $89.65 \pm 36.28$ & $94.47 \pm 42.42$ \\
Serum creatinine (g/dL) & $0.75 \pm 0.12$ & $0.78 \pm 0.15$ \\
eGFR (mL/min/1.73 m $\left.{ }^{2}\right)$ & & $89.6 \pm 14.5$ \\
Microalbuminuria (mg/24h) & & $9.38 \pm 6.67$ \\
Systolic blood pressure $(\mathrm{mmHg})$ & & $132.1 \pm 16.2$ \\
Diastolic blood pressure $(\mathrm{mmHg})$ & & $78.6 \pm 9.7$ \\
\hline
\end{tabular}

Data are mean \pm SD. eGFR $=$ estimated glomerular filtration rate calculated using the equation developed in the MDRD (Modification of Diet in Renal Disease) study.

Informed consent was obtained before enrolment. Institutional Review Board approval was obtained. The study was conducted in accordance with the ethical principles of the current Declaration of Helsinki.

2.2. Urinary GAG/PG Analysis. Urinary GAG/PG purification and quali-quantitative analysis were performed by a method previously described [12]. Briefly, early morning urine samples (about $50 \mathrm{~mL}$ ) were collected and, after centrifugation at $3,000 \times \mathrm{g}$ for 15 minutes at $4^{\circ} \mathrm{C}$, the sediment of broken cells or tissues and other solid materials was discarded. Clarified urine was applied to a column (EconoColumn Chromatography Columns, $0.5 \times 20 \mathrm{~cm}$, Bio-Rad Laboratories, CA, USA) packed with $6 \mathrm{~mL}$ of DEAE-Sephacel resin (GE Healthcare Life Sciences, UK), previously equilibrated with a buffer containing $0.02 \mathrm{M}$ tris- $\mathrm{HCl}, 0.15 \mathrm{M} \mathrm{NaCl}$ ( $\mathrm{pH}$ 8.6). After exhaustive washing, urinary GAGs/PGs were eluted with a buffer containing $0.02 \mathrm{M}$ tris- $\mathrm{HCl}, 2 \mathrm{M} \mathrm{LiCl}(\mathrm{pH}$ 8.6), and assayed for hexuronate content, using glucuronic acid as a standard. Hexuronate levels were normalized for urinary creatinine concentration, formerly determined by the Jaffè method (Sentinel CH, Milan, ITA). Urinary GAG/PG composition was determined by electrophoresis on cellulose acetate strips. The identification of GAGs/PGs was performed according to their comigration with standard GAGs and UTI purified from human urine (SCIPAC Ltd., UK) and to their electrophoretic profiles after enzymatic depolymerization with specific endoglycosidases. Images were acquired and analyzed by means of Gel Doc XR system and Quantity One software (Bio-Rad Laboratories, CA, USA). GAGs/PGs were expressed as relative percentages.

2.3. NAG and NGAL Determinations. Urinary NAG was estimated kinetically by using NAG colorimetric kit (FAR 

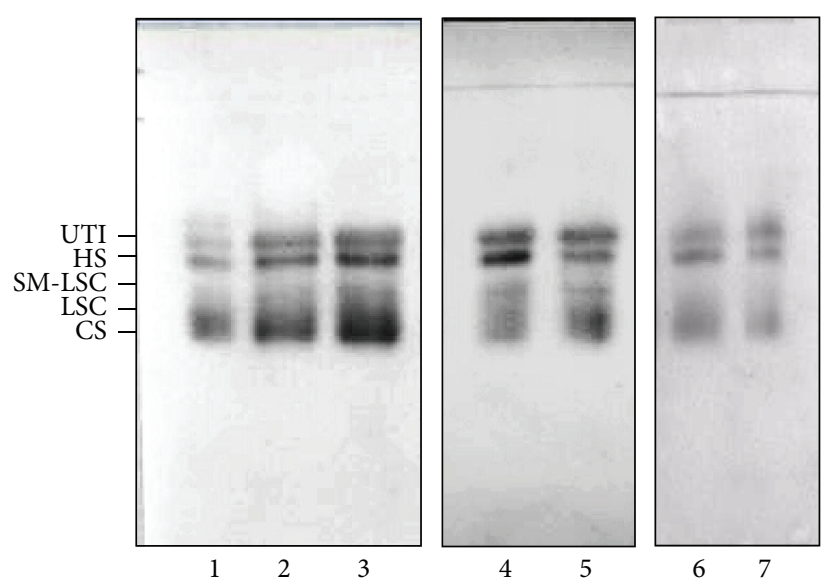

FigURE 1: Representative cellulose acetate electrophoretic profiles of urine glycosaminoglycans/proteoglycans from T2DM patients (lanes 1-5) and control subjects (lanes 6-7). UTI: urinary trypsin inhibitor. LSC: low sulfate chondroitin sulfate. SM-LSC: slow migration-LSC. HS: heparan sulfate. CS: chondroitin sulfate.

DIAGNOSTICS, Verona, ITA), whereas NGAL levels were performed with the Lipocalin-2/NGAL Human ELISA Kit (Abcam, Cambridge, UK). The assays were performed according to the protocols provided by the manufacturers.

2.4. Statistical Analysis. Statistical analyses were performed using the software package Sigma Stat 3 (Systat Software). Values are reported as mean $( \pm \mathrm{SD})$ or median (interquartile range). Differences between groups were assessed by Student's $t$-test or the Mann-Whitney rank sum test when data failed the normality test. Association between the examined variables was investigated nonparametrically by Spearman's rank correlation analysis. The statistical significance was set at $p<0.05$.

\section{Results}

The total urinary GAG excretion showed no significant differences between patients and controls (3.757 (2.868$5.234) \mathrm{mg}$ of hexuronate/g creatinine versus 4.40 (3.4755.635) $\mathrm{mg}$ of hexuronate/g creatinine, $p=0.201$ ). The electrophoretic profiles of urine GAG/PG samples (Figure 1) showed the presence of UTI and its degradation products (LSC and SM-LSC), CS, and HS. Following image analysis, a significant reduction of HS $(p<0.001$, Figure 2(a)) and a corresponding increase of total UTI (including UTI and its degradation products) $(p=0.034$, Figure 2(b)) were evidenced in patients, whereas no differences in CS percentage were found ( $p=0.272$, Figure 2(c)). The urinary excretion of NAG showed no differences between the two examined groups (3.547 (1.915-5.123) U/g creatinine versus $3.210(2.000-3.835) \mathrm{U} / \mathrm{g}$ creatinine, $p=0.202)$, whereas the median value of NGAL was significantly increased in T2DM patients with $p=0.025$ (Figure 3). The comparison among urinary levels of the evaluated markers and parameters such as age, BMI, duration of disease, and renal function indexes allowed us to detect significant relationships in T2DM patients among NGAL levels and both NAG $(r=$ 0.606, $p<0.0001)$ and the presence of hypertension $(r=$ $0.352, p<0.05)$.

\section{Discussion}

The identification of sensitive markers of renal function continues to be of considerable interest. Although UAE is considered the "gold standard" for diagnosing renal dysfunction, other markers, detectable at an earlier stage of the disease, may have diagnostic and/or prognostic value. Moreover, the association between glomerular lesions and MA is less pronounced in T2DM. In this respect, Fioretto et al. [13] found structurally normal glomeruli in approximately $30 \%$ of microalbuminuric T2DM patients, whereas they did not evidence MA in patients with glomerulosclerosis. The known correlation between MA and cardiovascular events [14] can be explained by the fact that both microvascular and macrovascular complications in T2DM are also present in the kidney, making MA an index of generalized vascular pathology.

Several studies have been performed to elucidate the molecular mechanisms underlying glomerular ultrafiltration, suggesting a role of PGs, especially their carbohydrate moieties, in maintaining the glomerular filtration barrier [15]. In this respect, the glycosaminoglycan moiety of HSPG is thought to be involved in the pathogenesis of diabetic nephropathy [16]. The presence of PGs such as perlecan [17] and agrin [18] and collagen VIII [19] in the GBM is well documented. These molecules contribute to the negative charge of the GBM and may thus play a role on the permselectivity of the glomerular barrier. However, a study on mice lacking exon 3 of perlecan (HSPG2) that causes loss of attachment sites for HS side chains did not detect any kidney impairment, such as proteinuria [20]. This could be due to compensatory mechanisms, but, most likely, this observation further strengthens the hypothesis that the GBM alone cannot be responsible for the permselective properties of the glomerular barrier. PGs are present on the surface of endothelial cells and podocytes, as well as in the mesangial matrix cells. Cultured immortalized human podocytes express both mRNA and protein for syndecan-1, versican, and perlecan [21]. The negative charges on podocytes are thought to contribute to charge selectivity, podocyte stability, and signaling. In this cross-sectional study, we observed a significant reduction of HS relative abundance in T2DM patients with respect to healthy subjects. This reduction may be related to an alteration of the processes of HS synthesis and/or sulfation. In this respect, Van Det et al. [22] described a reduction of both synthesis and sulfation of HSPG by mesangial cells and glomerular visceral epithelial cells treated with high doses of glucose. Moreover, Yokoyama et al. [23] reported a reduced HS N-sulfation in the urine of T2DM patients.

The alteration of tubular reabsorption is found almost always in the early stages of kidney disease, including those characterized by predominant glomerular involvement. In this study, we observed an increase of NGAL in 


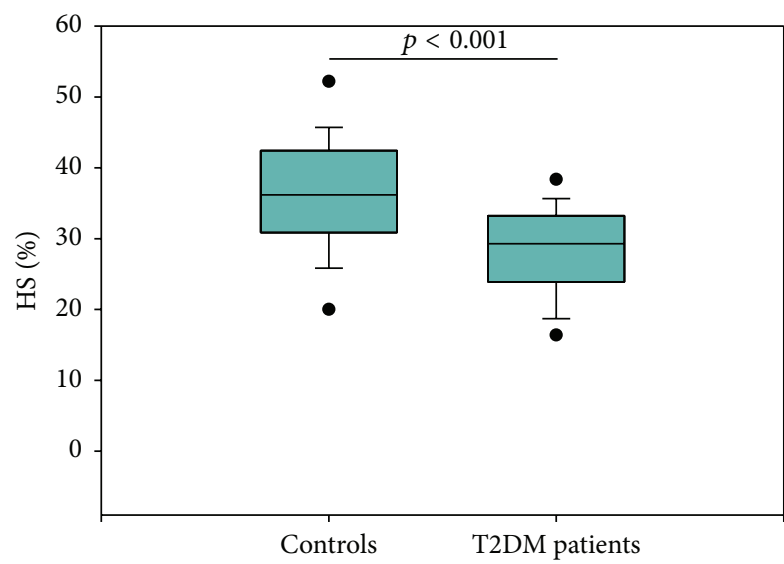

(a)

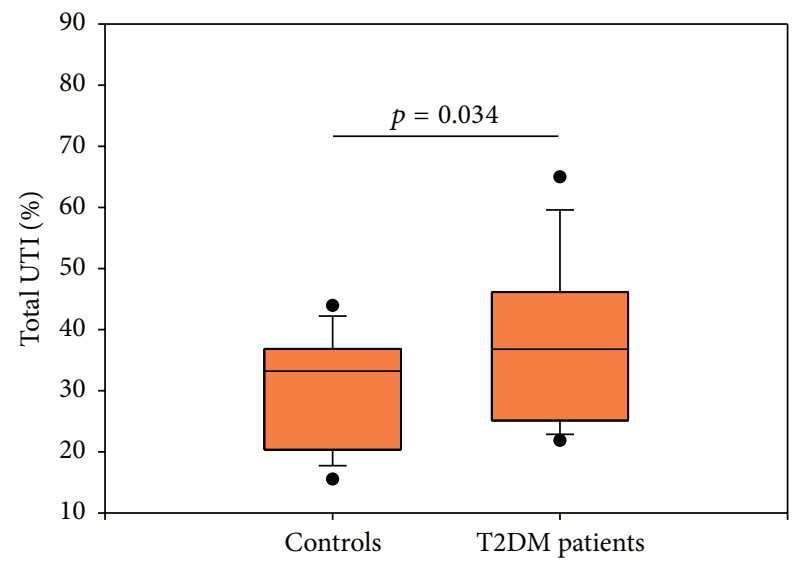

(b)

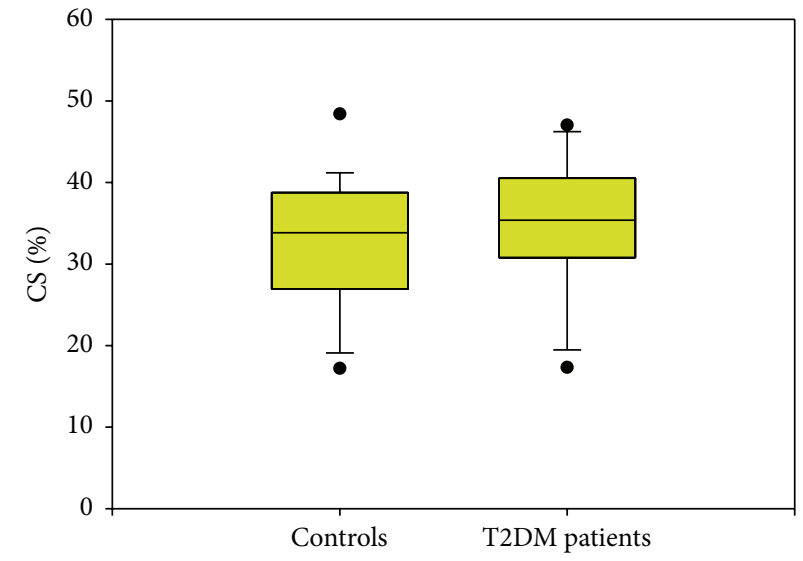

(c)

FIgURE 2: Plots showing the median (line within box), 25th and 75th percentiles (box), 5th and 95th percentiles (whiskers), and outliers $(\bullet)$ of HS (a), total UTI (UTI plus SM-LSC and LSC) (b), and CS (c) percentages in the controls group and in T2DM patients. Percentages were evaluated by performing image analysis on cellulose acetate electrophoretic profiles using Quantity One software (Bio-Rad Laboratories). Differences with $p$ values $<0.05$ were considered statistically significant.

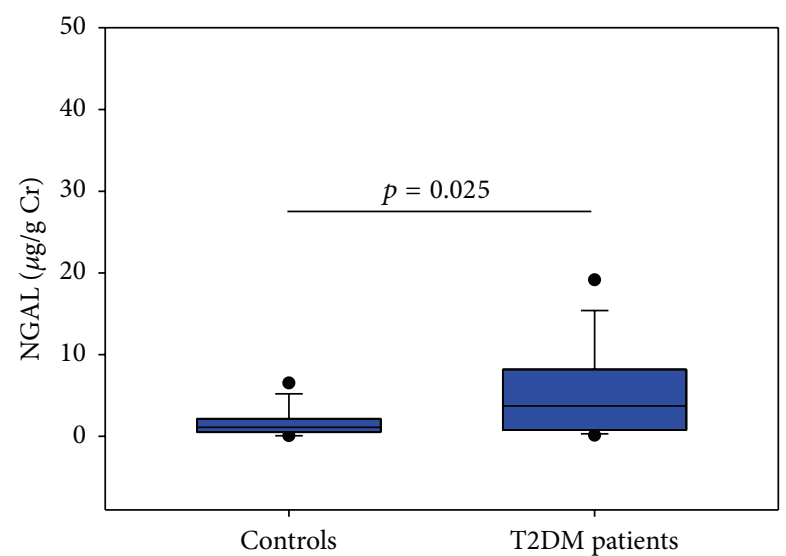

FIgURE 3: Plot showing the median (line within box), 25th and 75th percentiles (box), 5th and 95th percentiles (whiskers), and outliers $(\bullet)$ of neutrophil gelatinase-associated lipocalin (NGAL) levels, normalized for creatinine $(\mathrm{Cr})$ content, in the controls group and in T2DM patients. Differences with $p$ values $<0.05$ were considered statistically significant. normoalbuminuric diabetic patients. Being an iron transporter, NGAL may be expressed by the damaged renal tubule to induce regeneration, since this element is necessary for reepithelialization. Furthermore, it is known that pathological changes in diabetic nephropathy involve accumulation of extracellular matrix, the degradation of which involves mainly MMPs. The activities of these enzymes are dependent on metal ions and are limited by TIMP-1. In particular, it has been reported that a high glucose concentration causes a reduction in the amount of MMPs secreted by the mesangial cells [24] and an upregulated expression of TIMP-1 [25], leading to extracellular matrix accumulation. NGAL may represent a universal activator of the MMP family [26]. In fact, it is known that NGAL modulates MMP-9 activity by protecting it from degradation [27], activating directly the MMP-9 precursor, and counteracting the inhibiting effect of TIMP-1 [26]. Thus, it may be suggested that NGAL is overexpressed to delay the progression of renal fibrosis in diabetic nephropathy, by preserving the enzymatic activity of MMP-9. 
In our study, the urinary levels of NGAL show a highly significant relationship with the urinary NAG levels and a positive relationship with the presence of hypertension. We did not find any relationship among these parameters and serum creatinine levels or eGFR, probably because the increase of serum creatinine may not be evident before $50 \%$ or more of the nephrons are damaged.

Image analysis of GAG/PG profiles also enabled us to detect a significant increase in the relative content of total UTI in T2DM patients.

The role of inflammation in the pathogenesis of type 2 diabetes and associated complications is now well established. Multiple mechanisms underlie defective insulin secretion and responses in type 2 diabetes. These include glucotoxicity, lipotoxicity, oxidative stress, and endoplasmic reticulum stress. Interestingly, all of these mechanisms are associated with inflammatory responses [28].

During inflammation, UTI is released from $\mathrm{I} \alpha \mathrm{I}$ family proteins through proteolytic cleavage by neutrophil elastase in the peripheral circulation or at the inflammation site [29], therefore representing a positive acute phase protein. Furthermore, UTI is rapidly cleared from circulation by renal excretion into urine where it represents a useful inflammatory marker [7-9, 30].

In conclusion, we observed an increased level of NGAL, as well as an altered relative content of HS and total UTI in normoalbuminuric T2DM patients with significant correlations among NGAL levels and both NAG excretion and presence of hypertension. It is likely that, rather than a single biomarker, the identification of a panel of strategically selected analytes may represent a useful tool for the accurate prediction and monitoring of the disease. In this perspective, our results, although preliminary, suggest that the assessed markers could represent good candidates to detect early renal alteration in normoalbuminuric patients with T2DM. Further longitudinal studies with a larger sample size are required to clarify these results.

\section{Abbreviations}

T2DM: $\quad$ Type 2 diabetes mellitus

GAGs/PGs: Glycosaminoglycans/proteoglycans

NAG: $\quad$ N-Acetyl- $\beta$-D-glucosaminidase

NGAL: $\quad$ Neutrophil gelatinase-associated lipocalin

UTI: $\quad$ Urinary trypsin inhibitor

LSC: $\quad$ Low sulfate chondroitin sulfate

SM-LSC: Slow migration-LSC

HS: Heparan sulfate

CS: $\quad$ Chondroitin sulfate

ESRD: $\quad$ End-stage renal disease

CKD: $\quad$ Chronic kidney disease

UAE: Urinary albumin excretion

GBM: $\quad$ Glomerular basement membrane

BMI: $\quad$ Body mass index

MA: $\quad$ Microalbuminuria

HSPG: Heparan sulfate proteoglycan

MMPs: $\quad$ Matrix metalloproteinases

TIMP: $\quad$ Tissue inhibitor of metalloproteinase

eGFR: $\quad$ Estimated glomerular filtration rate.

\section{Conflict of Interests}

The authors declare that there is no conflict of interests regarding the publication of this paper.

\section{Acknowledgment}

This study was supported by Regione Autonoma della Sardegna (P.O.R. Sardegna F.S.E. 2007/2013, Asse IV Capitale Umano, Obiettivo Competitività Regionale e Occupazione, Asse IV Capitale Umano, Linea di Attività 1.3.1).

\section{References}

[1] World Health Organization, Prevention of Diabetes Mellitus, vol. 844 of Technical Report Series, World Health Organization, Geneva, Switzerland, 1994.

[2] M. I. Harris, R. Klein, T. A. Welborn, and M. W. Knuiman, "Onset of NIDDM occurs at least 4-7 yr before clinical diagnosis," Diabetes Care, vol. 15, no. 7, pp. 815-819, 1992.

[3] I. M. Stratton, E. M. Kohner, S. J. Aldington et al., "UKPDS 50: risk factors for incidence and progression of retinopathy in Type II diabetes over 6 years from diagnosis," Diabetologia, vol. 44, no. 2, pp. 156-163, 2001.

[4] M. J. Sarnak, A. S. Levey, A. C. Schoolwerth et al., "Kidney disease as a risk factor for development of cardiovascular disease: a statement from the American Heart Association Councils on Kidney in Cardiovascular Disease, High Blood Pressure Research, Clinical Cardiology, and Epidemiology and Prevention," Circulation, vol. 108, no. 17, pp. 2154-2169, 2003.

[5] J. L. Meyers, S. D. Candrilli, and B. Kovacs, "Type 2 diabetes mellitus and renal impairment in a large outpatient electronic medical records database: rates of diagnosis and antihyperglycemic medication dose adjustment," Postgraduate Medicine, vol. 123, no. 3, pp. 133-143, 2011.

[6] T. Deckert, B. Feldt-Rasmussen, R. Djurup, and M. Deckert, "Glomerular size and charge selectivity in insulin-dependent diabetes mellitus," Kidney International, vol. 33, no. 1, pp. 100106, 1988.

[7] P. De Muro, P. Fresu, M. Formato et al., "Urinary glycosaminoglycan and proteoglycan excretion in normoalbuminuric patients with type 1 diabetes mellitus," Journal of Nephrology, vol. 15, no. 3, pp. 290-296, 2002.

[8] A. J. Lepedda, G. Nieddu, S. Rocchiccioli, P. Fresu, P. De Muro, and M. Formato, "Development of a method for urine bikunin/ urinary trypsin inhibitor (UTI) quantitation and structural characterization: application to type 1 and type 2 diabetes," Electrophoresis, vol. 34, no. 22-23, pp. 3227-3233, 2013.

[9] A. J. Lepedda, L. Fancellu, E. Zinellu et al., "Urine bikunin as a marker of renal impairment in Fabry's disease," BioMed Research International, vol. 2013, Article ID 205948, 9 pages, 2013.

[10] R. E. Gilbert and M. E. Cooper, "The tubulointerstitium in progressive diabetic kidney disease: more than an aftermath of glomerular injury?" Kidney International, vol. 56, no. 5, pp. $1627-1637,1999$.

[11] D. Bolignano, G. Coppolino, A. Romeo et al., "Neutrophil gelatinase-associated lipocalin (NGAL) reflects iron status in haemodialysis patients," Nephrology Dialysis Transplantation, vol. 24, no. 11, pp. 3398-3403, 2009. 
[12] P. De Muro, R. Faedda, M. Formato et al., "Urinary glycosaminoglycans in patients with systemic lupus erythematosus," Clinical and Experimental Rheumatology, vol. 19, no. 2, pp. 125130, 2001.

[13] P. Fioretto, M. Mauer, E. Brocco et al., "Patterns of renal injury in NIDDM patients with microalbuminuria," Diabetologia, vol. 39, no. 12, pp. 1569-1576, 1996.

[14] H. C. Gerstein, J. F. E. Mann, Q. Yi et al., "Albuminuria and risk of cardiovascular events, death, and heart failure in diabetic and nondiabetic individuals," The Journal of the American Medical Association, vol. 286, no. 4, pp. 421-426, 2001.

[15] H. Morita, A. Yoshimura, and K. Kimata, "The role of heparan sulfate in the glomerular basement membrane," Kidney International, vol. 73, no. 3, pp. 247-248, 2008.

[16] T. Deckert, B. Feldt-Rasmussen, K. Borch-Johnsen, T. Jensen, and A. Kofoed-Enevoldsen, "Albuminuria reflects widespread vascular damage. The Steno hypothesis," Diabetologia, vol. 32, no. 4, pp. 219-226, 1989.

[17] R. V. Iozzo, I. R. Cohen, S. Grassel, and A. D. Murdoch, “The biology of perlecan: the multifaceted heparan sulphate proteoglycan of basement membranes and pericellular matrices," Biochemical Journal, vol. 302, no. 3, pp. 625-639, 1994.

[18] S. G. Hagen, A. F. Michael, and R. J. Butkowski, "Immunochemical and biochemical evidence for distinct basement membrane heparan sulfate proteoglycans," Journal of Biological Chemistry, vol. 268, no. 10, pp. 7261-7269, 1993.

[19] W. Halfter, S. Dong, B. Schurer, and G. J. Cole, "Collagen XVIII is a basement membrane heparan sulfate proteoglycan," The Journal of Biological Chemistry, vol. 273, no. 39, pp. 2540425412, 1998.

[20] M. Rossi, H. Morita, R. Sormunen et al., "Heparan sulfate chains of perlecan are indispensable in the lens capsule but not in the kidney," The EMBO Journal, vol. 22, no. 2, pp. 236-245, 2003.

[21] A. Björnson Granqvist, K. Ebefors, M. A. Saleem, P. W. Mathieson, B. Haraldsson, and J. Sörensson Nyström, "Podocyte proteoglycan synthesis is involved in the development of nephrotic syndrome," The American Journal of PhysiologyRenal Physiology, vol. 291, no. 4, pp. F722-F730, 2006.

[22] N. F. Van Det, J. Van Den Born, J. T. Tamsma et al., "Effects of high glucose on the production of heparan sulfate proteoglycan by mesangial and epithelial cells," Kidney International, vol. 49, no. 4, pp. 1079-1089, 1996.

[23] H. Yokoyama, K. Sato, M. Okudaira et al., "Serum and urinary concentrations of heparan sulfate in patients with diabetic nephropathy," Kidney International, vol. 56, no. 2, pp. 650-658, 1999.

[24] S. V. McLennan, D. K. Yue, and J. R. Turtle, "Effect of glucose on matrix metalloproteinase activity in mesangial cells," Nephron, vol. 79, no. 3, pp. 293-298, 1998.

[25] E. Ishimura, Y. Nishizawa, S. Shoji, and H. Morii, "Serum type III, IV collagens and TIMP in patients with type II diabetes mellitus," Life Sciences, vol. 58, no. 16, pp. 1331-1337, 1996.

[26] M. Coles, T. Diercks, B. Muehlenweg et al., "The solution structure and dynamics of human neutrophil gelatinase-associated lipocalin," Journal of Molecular Biology, vol. 289, no. 1, pp. 139157, 1999.

[27] L. Yan, N. Borregaard, L. Kjeldsen, and M. A. Moses, “The high molecular weight urinary matrix metalloproteinase (MMP) activity is a complex of gelatinase B/MMP-9 and neutrophil gelatinase-associated lipocalin (NGAL). Modulation of MMP-9 activity by NGAL," The Journal of Biological Chemistry, vol. 276, no. 40, pp. 37258-37265, 2001.
[28] M. Y. Donath, "Targeting inflammation in the treatment of type 2 diabetes: time to start," Nature Reviews Drug Discovery, vol. 13, no. 6, pp. 465-476, 2014.

[29] M. Balduyck, D. Albani, M. Jourdain et al., "Inflammationinduced systemic proteolysis of inter-alpha-inhibitor in plasma from patients with sepsis," Journal of Laboratory and Clinical Medicine, vol. 135, no. 2, pp. 188-198, 2000.

[30] M. Balduyck and J. Mizon, "Inter-alpha-trypsin inhibitor and its plasma and urine derivatives," Annales de Biologie Clinique, vol. 49, pp. 273-281, 1991. 

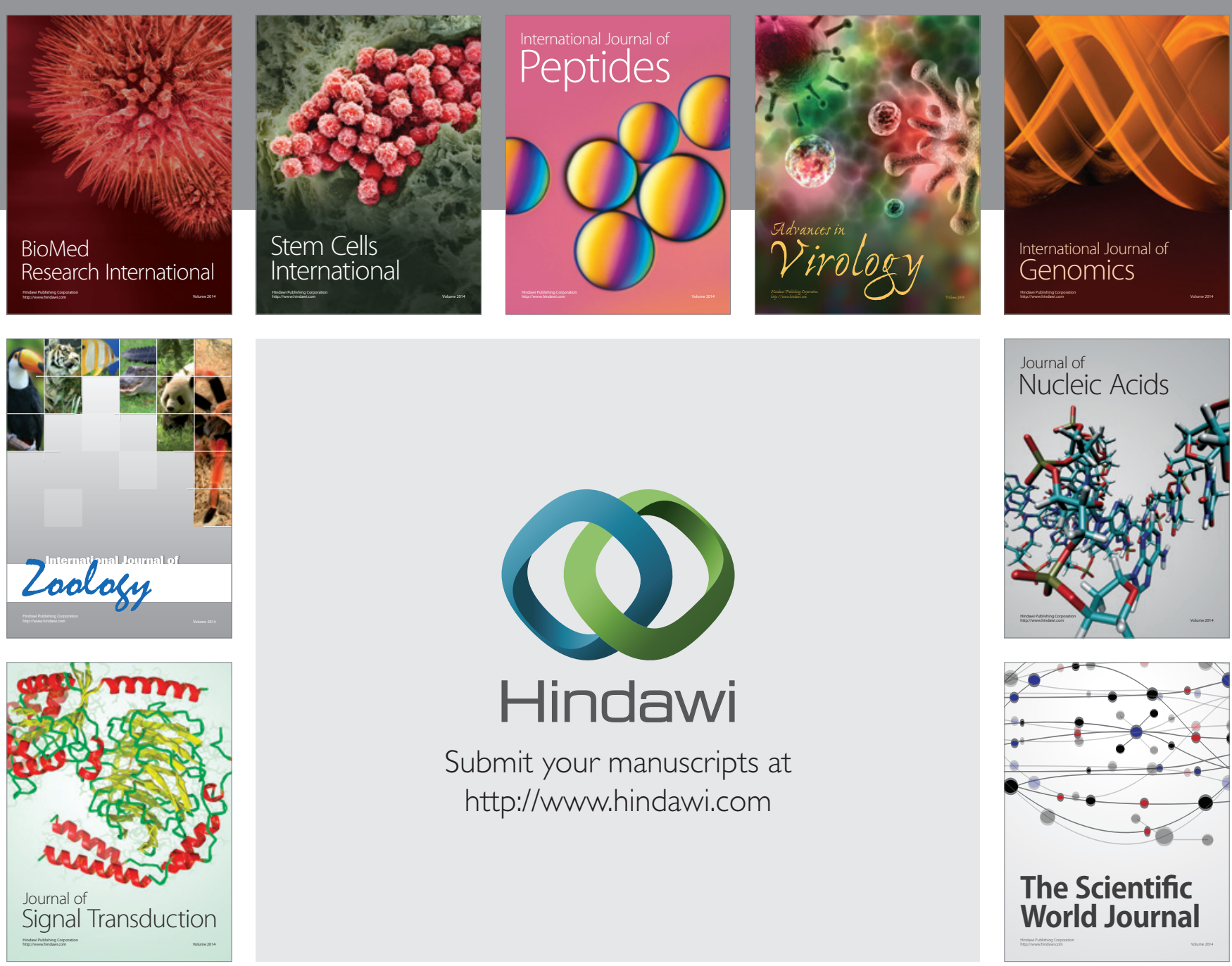

Submit your manuscripts at

http://www.hindawi.com
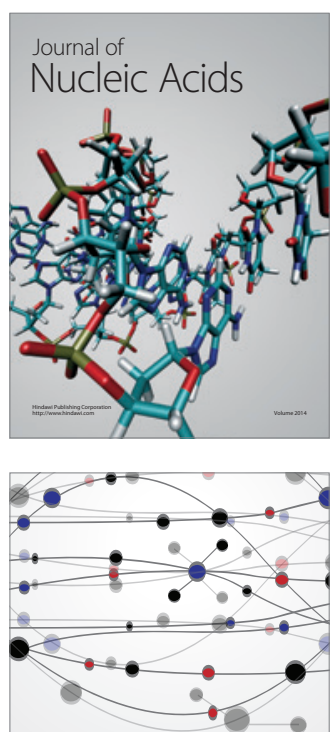

The Scientific World Journal
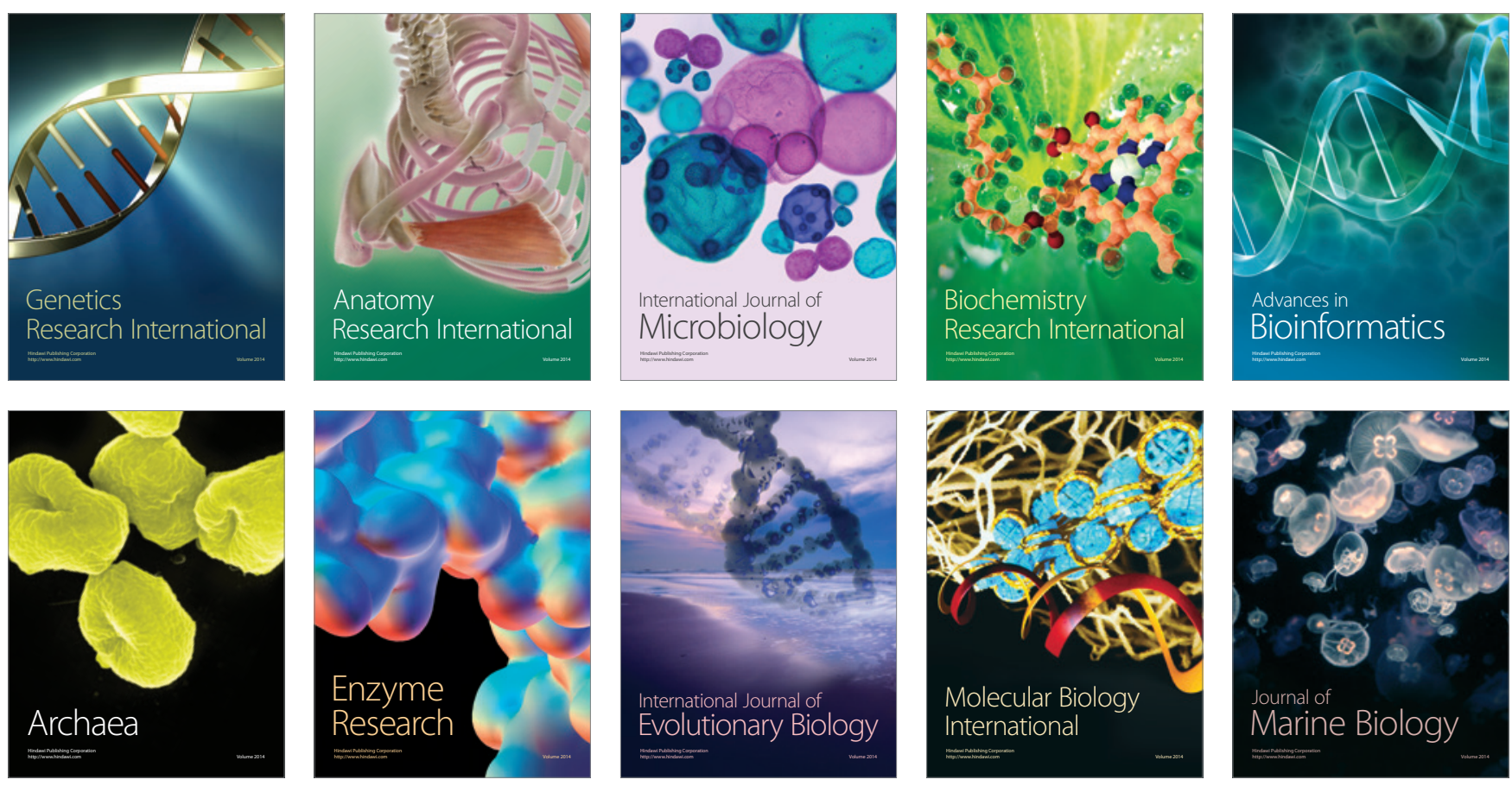